\title{
AC 2007-2183: THE INTEND EDUCATION PROGRAM: A NEW MODEL FOR MULTIDISCIPLINARY, DISPERSED EDUCATION
}

Timothy Hinds, Michigan State University

John Lloyd, Michigan State University 


\section{The INTEnD Education Program A New Model for Multidisciplinary, Dispersed Education}

\section{Introduction}

Since 1998, engineering, cultural anthropology and telecommunications faculty at Michigan State University and several other universities around the world have collaborated to develop a multidisciplinary engineering design program for the purpose of studying and practicing how internationally-dispersed engineering product design teams may function more effectively ${ }^{1,2}$.

The INTEnD (International Networked Teams for Engineering Design) program has studied and taught students of several collaborating disciplines in universities around the world about the critical barriers that leadership, power, trust, language, time, cultural, communication and educational differences pose to dispersed teams in addition to the many technical issues the engineering design process normally poses on a design team ${ }^{3,4}$.

\section{Globalization and the Engineer of 2020}

In 2003, the National Academy of Engineering published the report "The Engineer of 2020; Visions of Engineering in the New Century"5. It defines the concept of requirements for engineers beyond basic fundamentals; beyond current, traditional courses. That is, the need to evolve traditional classroom courses into dispersed virtual learning experiences.

Much of the motivation for writing such a report hinges on globalization. Without the current status of globalization, the report would not have been written. The process of globalization has created an environment where we must do more for engineering education to influence the US economy such that the United States is able to retain its leadership position.

Globalization will drive more uniformity in the educational systems between that of the United States and those around the world. We will require additional knowledge to remain globally competitive and maintain that global leadership.

Globalization also levels the playing field for competitiveness. For both industry and education, it redefines societal, geopolitical, and technical contexts of how engineers function. Thus, globalization drives the necessity for revolution of our educational system. This applies not only to engineering, but to all fields of professional study.

\section{Capabilities of 21st Century Professionals}

As defined in the "Engineer of 2020" report, engineers must not only possess strong analytic skills, but must also have the ability to communicate effectively. For engineers to be successful, that communication must span diverse cultures, languages, and disciplines. The new professionals we are educating and will educate must also be the drivers of innovation. This differs greatly from the old business processes we are used to and are comfortable employing. 
Much of the current professional coursework focuses on management of various technical aspects of business. To compete globally requires a strong understanding of the global business goals as well as the ability to lead dispersed, multidiscipline teams. That leadership requires cultural understanding as well as a strong global business understanding. It requires more than just business management.

Let us examine the differences between management and leadership. The term management is commonly used to define a "person or persons controlling and directing the affairs of a business" . Whereas leadership is "an act or instance of leading, guiding or direction" 6 . Managers do not have to be good leaders. They just need to have control. But, leaders must have good management skills to effectively guide and direct.

Similarly, we may examine the terms training and education. Training has been defined as "the education, instruction, or discipline of a person or thing that is being trained"6. Conversely, education may be defined as "the act or process of imparting or acquiring general knowledge, developing the powers of reasoning and judgment, and generally of preparing oneself or others intellectually for mature life."

To educate engineering professionals for practice in the 21 st century, we must set high goals while agreeing on an educational vision. The goals must be exciting to implement a change in the transformation of engineering education. We must also accommodate educational innovations from non-engineering fields. For the new vision to succeed, we must focus multidiscipline professionals on technical, social, and governance goals. The creation of new mindsets among faculty is critical to achieve these new educational goals. In the classroom, we must have a clear vision for our graduates to succeed.

\section{Implementation}

How do we make this change in engineering education happen? First, we need to understand that we must retain the core fundamental engineering material. These fundamentals must include those of all the key professions.

But, we must also integrate contextual fundamentals from key enabling professions. To have multi-cultural aspects in our new professionals, we must have representation from those in cultural education. If business aspects are important to our success, then our educational model must include business professionals. Likewise, it must also include communications and any other profession key to our success. This integration is the INTEnD Education Program goal.

Current educational strategies typically take one of two paths. The first employs separate courses taught independently in appropriate departments. If business aspects are needed in the educational model, students take a business course. The same applies for technical topics, anthropological issues, etc. The students take separate courses taught by different professors.

The second model consists of all of the topics covered by a single professor who is expert in the prime technology and has limited knowledge of the enabling topics. This situation is not optimal 
as it is very rare to have a single person who is well versed in all of the necessary topics. Often, the result is incomplete information and training being passed on to the student.

\section{The INTEnD Model}

The INTEnD model integrates knowledge from the enabling disciplines into the key discipline. This integration of courses can be local or distance/virtual. We utilize industry-driven collaborative projects to provide the focus of learning.

The core discipline in the INTEnD educational model is technology. However, to be successful, we must also teach students something about culture, communication, teamwork, leadership, logistics and innovation. Figure 1 illustrates the INTEnD model with the sharing of knowledge across the boundaries representing the total education experience. Together, these disciplines create the total education experience.

Full, semester-long courses usually are not required to educate students in each of the various required disciplines. The challenge is in assembling just enough instructional material to sufficiently transfer the required knowledge. In the INTEnD model, each discipline is taught by a professional in that particular field. The INTEnD Education model result is expert instruction in all key disciplines along with knowledge sharing across discipline boundaries.

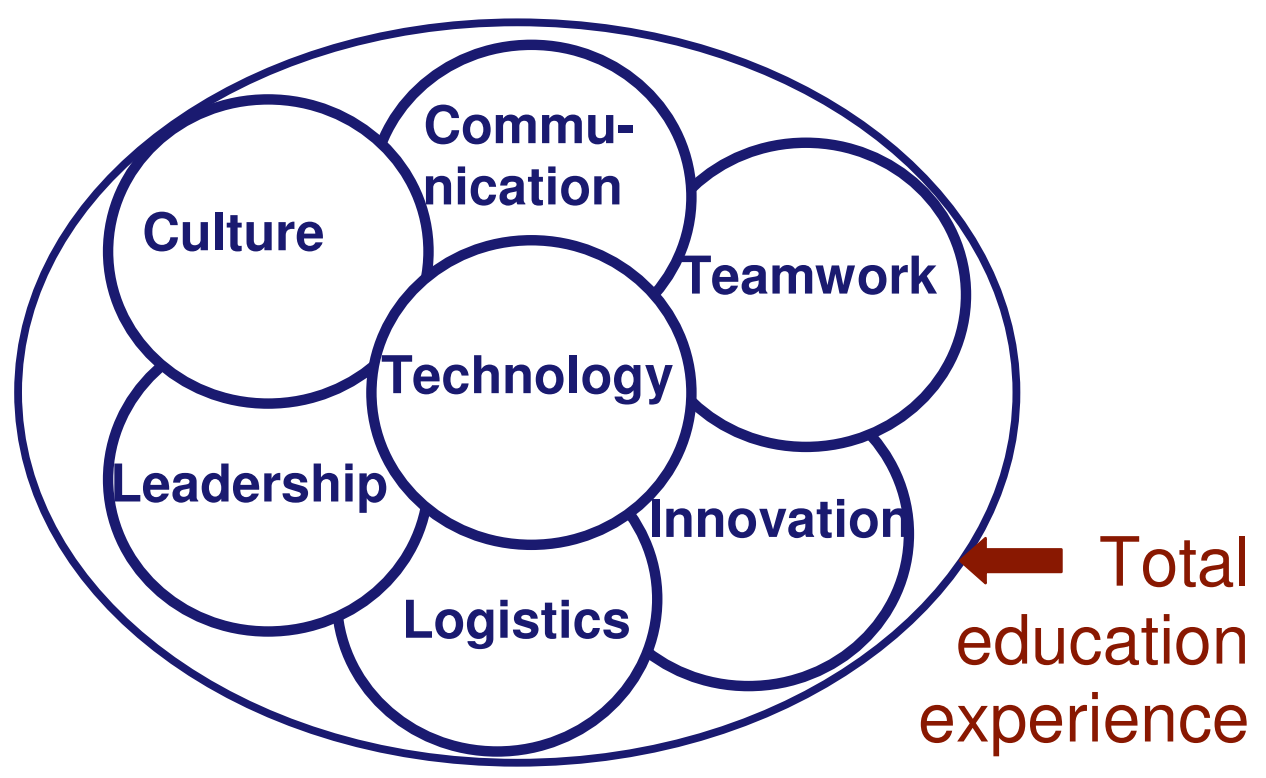

Figure 1: The INTEnD Educational Model

\section{The 21st Century Professional}

In the INTEnD model, engineering, anthropology and telecommunications share the classroom. In fact, engineering includes the disciplines of mechanical engineering, manufacturing engineering, and industrial engineering, as they are all necessary in the development of new, 
global products. The model includes an industrially sponsored project focusing teams of students onto single, coordinated tasks. Figure 2 below illustrates how we have built the model for the new engineer as collaboration between engineering, cultural anthropology and telecommunications to educate students from the three disciplines to break the barriers imposed on multi-national, multi-cultural, multi-discipline and boundary spanning teams. The figure may be understated, but there is great significance to be gleaned from the collaboration of professionals to accomplish education of the new $21^{\text {st }}$ century professional. Collaborations across nations, across cultures, across disciplines and across boundaries will allow any professional to work effectively in the global environment.

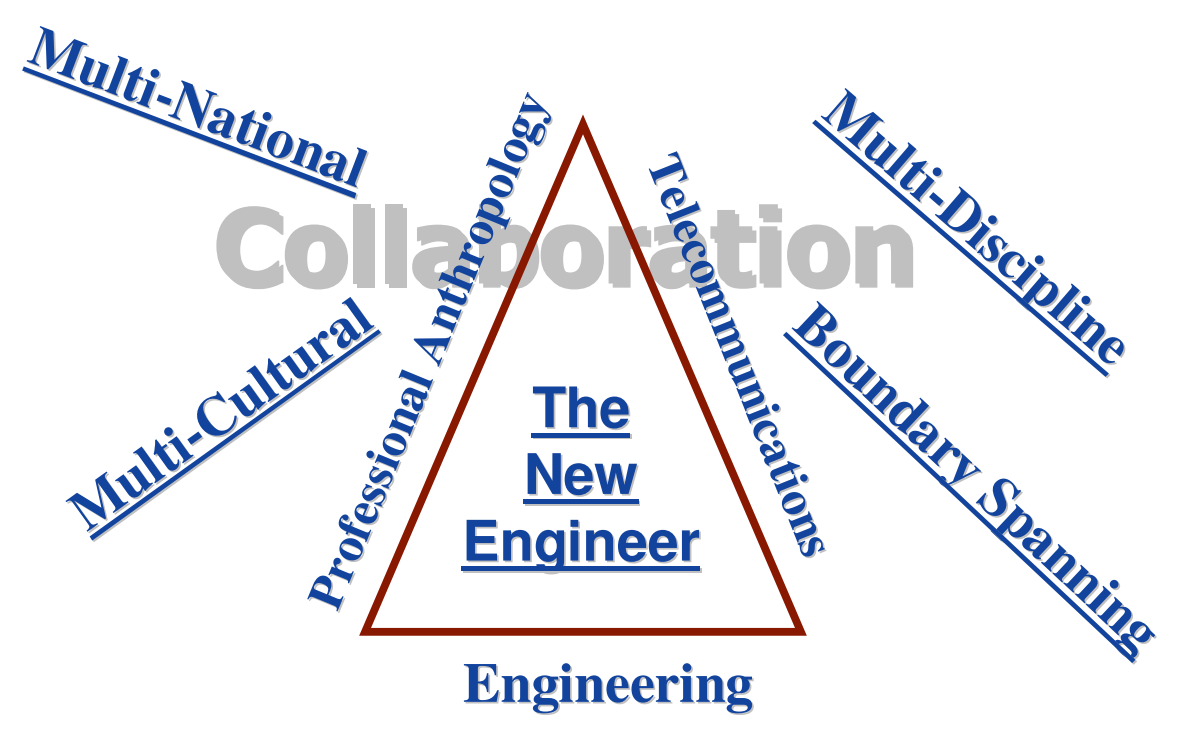

Figure 2: The $21^{\text {st }}$ Century Professional

Why have the various engineering students in the same classroom as the cultural anthropology and telecommunications students? The answer is that they educate each other in the key fundamentals of their respective disciplines. Their collaborative teamwork experience broadens their leadership, innovation, and communications learning experience. The experience is true to "real" life.

Knowledge and engineering have become commodities in many organizations, mostly due to the lack of differentiation in education and resulting capabilities. This is evidenced by outsourcing and off-shoring of technical work to obtain global advantage. Outsourcing and off-shoring tend only to be cost issues and not capability issues. An example of this practice is the radiological interpretation of $\mathrm{x}$-rays. The $\mathrm{x}$-rays may be read locally or read in another country with the information forwarded back to the source. In the latter case, the task may be completed in same amount of time but at a lower cost. 
The INTEnD model of education increases the personal competitiveness of the student in the global job market and makes them more than just a commodity. The model drives more than just a low cost commodity.

The INTEnD educational concept is not only for engineering education. The concept may be applied to any field of education. It simply requires the identification of the core plus the contextual education necessary to take a professional to be above and beyond a commodity.

\section{Conclusions}

The new INTEnD Education Program has taken a further step in the revolution brought on by distributed, global, multidiscipline engineering education by developing a new model that is applicable not only to engineering teams, but to any teams conducting distributed, multidisciplinary education activities. The classroom in the INTEnD Education Program model brings several disciplines together so that a "hands-on" collaborative experience is provided to help educate the participants about the several disciplines represented. For example, the engineer learns about leadership and product use in a multicultural environment by studying along side a companion Cultural Anthropology class. The class learns about communications though the inclusion of a companion course in Telecommunications. They all learn about the role of business strategy by the inclusion of a companion business course in the same classroom. Each college/department retains control over their own segment of the class, while the global, multidisciplinary learning aspects necessary for tomorrow's graduates are provided in a single classroom setting.

Finally, it is not necessary that the multiple disciplines all be in the same university. Through use of the "virtual" classroom, dispersed teaming can also be taught. The INTEnD classroom is the key to drive the co-learning needs for the workforce of tomorrow. The INTEnD Education Program provides the global, multidisciplinary education in a single, virtual classroom. This is an innovative, efficient and effective approach to providing the education that will best prepare the next generation workforce. INTEnD is a revolution in the education model of the $21 \mathrm{st}$ century professional.

\section{Bibliography}

1 "INTEnD: A Dispersed Design Team Approach for the Globalization of Engineering Education," American Society of Mechanical Engineers 2004 Curriculum Innovation Award Honorable Mention, November 2004, Anaheim, CA; J. Lloyd, T. Hinds, K. David, M. Chung, M. Gonzalez and D. Timmer.

2 "New Methods for Studying Global Virtual Teams: Towards a Multi-Faceted Approach," Copyright 2001 IEEE, published in the Proceedings of the Hawaii International Conference On System Sciences, January 3-6, 2001, Maui, Hawaii; C. Steinfield, M. Huysman, K. David, C. Jang, J. Poot, M. Veld, I. Mulder, E. Goodman, J. Lloyd, T. Hinds, E. Andriessen, K. Jarvis, K. van der Werff, and A. Cabrera.

3 "Virtual Teams: Communicating in a Global Business," Proceedings of 2001 ASME International Mechanical Engineering Conference and Exposition, November 11-16, 2001, New York, NY, IMECE2001/T\&S-23405; C. A. Schlueter, J. R. Lloyd, T. J. Hinds and E. D. Goodman. 
4 "Power and Communication: Solving Power Problems for Collaboration in Globally Distributed Engineering Design Teams," IMECE2005-80060, 2005 ASME International Mechanical Engineering Congress and RD\&D Expo, November 5-11, 2005, Orlando, FL, K. David, J. Lloyd and T. Hinds.

5 "The Engineer of 2020, Visions of Engineering in the New Century," National Academy of Engineering, The National Academies Press, 2003.

${ }^{6}$ www.dictionary.com. 